\title{
Negative values of cosine sums
}

\author{
by
}

Imre Z. Ruzsa (Budapest)

1. Introduction. Let $A$ be a finite set of positive integers, $|A|=n$, and write

$$
f(x)=\sum_{a \in A} \cos a x .
$$

Since $f(0)>0$ and $\int_{0}^{2 \pi} f(x) d x=0$, we have $\min f(x)<0$. It is a difficult question to estimate this minimum uniformly for every set of size $n$. Bourgain [2] proved

$$
\min f(x)<-c_{1} e^{-c_{2}(\log n)^{c_{3}}}
$$

with unspecified positive constants $c_{1}, c_{2}, c_{3}$. In another paper [1] he showed that one can take $c_{3}=1 / 2$ under the assumption that $A \subset\left[1, n 2^{\sqrt{\log n}}\right]$. Our aim is to prove this without restriction.

THEOREM 1. With the above notations we have

$$
\min f(x)<-c_{4} e^{-c_{5} \sqrt{\log n}}
$$

with a positive absolute constant $c_{4}$ and $c_{5}=\sqrt{(\log 2) / 8}$.

First we give a reformulation with exponentials, as this form will be more convenient to work with. We write $e(x)=e^{2 \pi i x}$.

TheOREM 2. Let $A$ be a finite set of integers, symmetric about the origin and not containing 0 . Put

With $|A|=n$ we have

$$
f(x)=\sum_{a \in A} e(a x)
$$

$$
\min f(x)<-c_{4} e^{-c_{5} \sqrt{\log n}}
$$

with a positive absolute constant $c_{4}$ and $c_{5}=\sqrt{(\log 2) / 8}$.

2000 Mathematics Subject Classification: 11B75, 11L99, 42A05.

Supported by Hungarian National Foundation for Scientific Research, grants no. T 25617 and T 29759. 
(The number $n$ and the function $f$ in Theorem 2 are twice the corresponding quantities of Theorem 1.)

In the first stage of the proof (Section 2) we find a regular subset of $A$ under the assumption that $K$ is small. In the second stage (Section 3 ) we show that the existence of a regular subset yields a bound on this minimum. The synthesis and a comparison to Bourgain's paper is given in Section 4 .

\section{From minimum to structure}

Lemma 2.1. Let $A$ be a finite set of integers, symmetric about the origin and not containing 0 . Let

$$
f(x)=\sum_{a \in A} e(a x) .
$$

Write $|A|=n, \min f(x)=-K$ and

$$
k=\left[\frac{\log N}{4 \log K+c_{6}}\right]
$$

with a suitable absolute constant $c_{6}$. There are integers $\beta_{1}, \ldots, \beta_{k}$ and a set $B \subset \mathbb{Z}$ such that

$$
B+\left\{\sum \varepsilon_{i} \beta_{i}: \varepsilon_{i} \in\{0,1\}\right\} \subset A,
$$

the $2^{k}$ sums

$$
\left\{\sum \varepsilon_{i} \beta_{i}: \varepsilon_{i} \in\{0,1\}\right\}
$$

are all distinct and $|B| \geq \sqrt{n}$.

Proof. We shall find inductively integers $\beta_{1}, \ldots, \beta_{k}$ and sets of integers $B_{0}, B_{1}, \ldots, B_{k}$ with the following properties for every $j \leq k$. First, the $2^{j}$ numbers $\sum_{\nu=1}^{j} \varepsilon_{\nu} \beta_{\nu}, \varepsilon_{\nu} \in\{0,1\}$, are all distinct. Next, we always have

$$
\begin{gathered}
\sum_{\nu=1}^{j} \varepsilon_{\nu} \beta_{\nu}+B_{j} \subset A, \\
\left|B_{j}\right| \geq M_{j}=\left(4 K^{2}\right)^{-j} n .
\end{gathered}
$$

The last property asserts that the function

$$
g_{j}(x)=\sum_{b \in B_{j}} e^{2 \pi i b x}
$$

has a decomposition $g_{j}=h_{1}^{(j)}+h_{2}^{(j)}+h_{3}^{(j)}$ such that

$$
\begin{aligned}
\left|h_{1}^{(j)}(x)\right| & \leq f(x)+K \quad \text { for all } x, \\
\left|h_{2}^{(j)}(x)\right| & \leq L_{j}=4^{j} K^{j+1} \quad \text { for all } x, \\
\left\|h_{3}^{(j)}\right\|_{1} & \leq \eta_{j}=\left(8 K^{2}\right)^{j} n^{-1 / 2} .
\end{aligned}
$$


An important consequence of (2.6) is that

$$
\left\|h_{1}^{(j)}\right\|_{1} \leq\|f+K\|_{1}=K .
$$

We start with $B_{0}=A$. The above decomposition of $g_{0}=f$ will be

$$
\begin{aligned}
h_{1}^{(0)}(x) & =f(x)^{+}=\max (0, f(x)), \\
h_{2}^{(0)}(x) & =-f(x)^{-}, \quad L_{0}=K, \\
h_{3}^{(0)} & =0 .
\end{aligned}
$$

Assume now that the set $B_{j}$, the integers $\beta_{1}, \ldots, \beta_{j}$ and the functions $h_{\nu}^{(j)}$ are given. We are going to find $B_{j+1}$ and the functions $h_{\nu}^{(j+1)}$. To simplify notation we shall write $B, M, g, h_{\nu}, L, \eta$ for $B_{j}, M_{j}, g_{j}, h_{\nu}^{(j)}, L_{j}, \eta_{j}$, and $B^{\prime}, M^{\prime}, g^{\prime}, h_{\nu}^{\prime}, L^{\prime}, \eta^{\prime}$ for $B_{j+1}, M_{j+1}, g_{j+1}, h_{\nu}^{(j+1)}, L_{j+1}, \eta_{j+1}$.

Write $|B|=m(\geq M)$. We will seek $B^{\prime}$ in the form $B^{\prime}=B \cap(B-\alpha)$, and then put $\beta_{j+1}=\alpha$. This guarantees (2.4).

To estimate the size of such an intersection, first observe that

$$
\begin{aligned}
& \sum_{\alpha \in \mathbb{Z}}|B \cap(B-\alpha)|=m^{2}, \\
& \sum_{\alpha \in \mathbb{Z}}|B \cap(B-\alpha)|^{2}=\|g\|_{4}^{4} .
\end{aligned}
$$

To estimate this quantity we start with

$$
\begin{aligned}
& \left\|g-h_{2}\right\|_{2} \geq\|g\|_{2}-\left\|h_{2}\right\|_{2} \geq\|g\|_{2}-\left\|h_{2}\right\|_{\infty} \geq \sqrt{m}-L, \\
& \left\|g-h_{2}\right\|_{1}=\left\|h_{1}+h_{3}\right\|_{1} \leq K+\eta,
\end{aligned}
$$

by (2.6) and (2.9). By Hölder's inequality we have

$$
\begin{aligned}
\left\|g-h_{2}\right\|_{4} & \geq \frac{\left\|g-h_{2}\right\|_{2}^{3 / 2}}{\left\|g-h_{2}\right\|_{1}^{1 / 2}} \geq \frac{(\sqrt{m}-L)^{3 / 2}}{(K+\eta)^{1 / 2}} \\
& \geq \frac{8}{9} m^{3 / 4} K^{-1 / 2}
\end{aligned}
$$

if we suppose

$$
\eta<c K, \quad L<c \sqrt{M} \leq c \sqrt{m}
$$

with a suitably small positive constant $c$. This implies

$$
\|g\|_{4} \geq\left\|g-h_{2}\right\|_{4}-\left\|h_{2}\right\|_{4} \geq \frac{8}{9} m^{3 / 4} K^{-1 / 2}-L \geq \frac{7}{8} m^{3 / 4} K^{-1 / 2}
$$

if $L \sqrt{K}<\mathrm{cm}^{3 / 4}$. This assumption follows from the second inequality of (2.12), since $L \geq K$ by (2.7). 
By (2.11) we obtain

$$
\sum_{\alpha \in \mathbb{Z}}|B \cap(B-\alpha)|^{2} \geq \frac{1}{2} \frac{m^{3}}{K^{2}} .
$$

The contribution of terms satisfying $|B \cap(B-\alpha)| \leq \frac{1}{4} m / K^{2}$ to this sum is at most $\frac{1}{4} m^{3} / K^{2}$ by $(2.10)$, so at least $\frac{1}{4} m^{3} / K^{2}$ comes from $\alpha$ such that $|B \cap(B-\alpha)|>\frac{1}{4} m / K^{2}$. As each summand is at most $m^{2}$, we infer that there are $>\frac{1}{4} m / K^{2}$ values of $\alpha$ such that $|B \cap(B-\alpha)|>\frac{1}{4} m / K^{2}$.

We shall select our $\beta_{j}=\alpha$ from these values. This guarantees the inductive step for (2.5). In order that the sums $\sum_{\nu=1}^{j+1} \varepsilon_{\nu} \beta_{\nu}$ be distinct, we need to avoid the at most $3^{j}$ numbers of the form

$$
\sum_{\nu=1}^{j} \delta_{\nu} \beta_{\nu}, \quad \delta_{\nu} \in\{-1,0,1\} .
$$

If we suppose

$$
3^{j} \leq \frac{m}{8 K^{2}}
$$

then we still have $\geq m /\left(8 K^{2}\right)$ values of $\beta$ to choose from.

We have to find the decomposition of $g^{\prime}$ and show properties (2.6)-(2.8).

Write $e_{\alpha}(x)=e^{2 \pi i \alpha x}$. With this notation we can write $g^{\prime}$ as a convolution

$$
g^{\prime}=g * g e_{\alpha} .
$$

If we substitute the decomposition of $g$ into this formula we get an expression for $g^{\prime}$ as a sum of 9 convolutions, which will be dealt with in different ways.

First observe that

$$
\left|h_{1} * h_{1} e_{\alpha}\right| \leq\left|h_{1}\right| *\left|h_{1}\right| \leq(f+K) *(f+K)=f+K^{2}
$$

(in the sense that this inequality holds for every value of the variable). In the last step we use the fact that $f * f=f$, which is equivalent to the property that each coefficient is 0 or 1 .

Clearly we can decompose $h_{1} * h_{1} e_{\alpha}$ as

$$
h_{1} * h_{1} e_{\alpha}=h_{1}^{\prime}+h_{21}^{\prime},
$$

where $\left|h_{1}^{\prime}\right| \leq f+K$ and $\left|h_{21}^{\prime}\right| \leq K^{2}-K$. The function $h_{21}^{\prime}$ will contribute to $h_{2}^{\prime}$.

Other contributions to $h_{2}^{\prime}$ come from convolutions involving $h_{2}$ and $h_{1}$ or $h_{3}$. We have

$$
\left\|\left(h_{1}+h_{3}\right) * h_{2} e_{\alpha}\right\|_{\infty} \leq\left\|h_{1}+h_{3}\right\|_{1}\left\|h_{2}\right\|_{\infty} \leq(K+\eta) L
$$

and the same estimate holds for $\left\|\left(h_{1}+h_{3}\right) e_{\alpha} * h_{2}\right\|_{\infty}$. So finally

$$
h_{2}^{\prime}=h_{21}^{\prime}+\left(h_{1}+h_{3}\right) * h_{2} e_{\alpha}+\left(h_{1}+h_{3}\right) e_{\alpha} * h_{2}
$$


satisfies

$$
\left\|h_{2}^{\prime}\right\|_{\infty} \leq\left(K^{2}-K\right)+2(K+\eta) L \leq 4 K L .
$$

This is exactly inequality (2.7) for $j+1$.

The other terms make up $h_{3}^{\prime}$. We have

$$
\left\|h_{1} * h_{3} e_{\alpha}\right\|_{1} \leq\left\|h_{1}\right\|_{1}\left\|h_{3}\right\|_{1} \leq \eta K,
$$

and the same estimate holds for $\left\|h_{3} * h_{1} e_{\alpha}\right\|_{1}$. Similarly

$$
\left\|h_{3} * h_{3} e_{\alpha}\right\|_{1} \leq\left\|h_{3}\right\|_{1}^{2} \leq \eta^{2}<\eta K .
$$

For the estimation of $\left\|h_{2} * h_{2} e_{\alpha}\right\|_{1}$ we shall use averaging in $\alpha$. An application of Parseval's formula yields

$$
\sum_{\alpha \in \mathbb{Z}}\left\|h_{2} * h_{2} e_{\alpha}\right\|_{2}^{2}=\left\|h_{2}\right\|_{2}^{4} \leq\left\|h_{2}\right\|_{\infty}^{4} \leq L^{4} .
$$

Since we have at least $m /\left(8 K^{2}\right)$ values of $\alpha$ to choose from, there is one such that

$$
\left\|h_{2} * h_{2} e_{\alpha}\right\|_{1} \leq\left\|h_{2} * h_{2} e_{\alpha}\right\|_{2}<3 K L^{2} m^{-1 / 2}
$$

So

$$
h_{3}^{\prime}=h_{1} * h_{3} e_{\alpha}+h_{3} * h_{1} e_{\alpha}+h_{3} * h_{3} e_{\alpha}+h_{2} * h_{2} e_{\alpha}
$$

satisfies

$$
\left\|h_{3}^{\prime}\right\|_{1}<3 \eta K+3 K L m^{-1 / 2} .
$$

By substituting the definition of $\eta$ and $L$ from (2.8), (2.7) and using the lower estimate (2.5) for $m$ a simple calculation shows $(2.8)$ for $j+1$. This $\alpha$ will be our $\beta_{j+1}$, and this ends the induction.

This process goes on as long as conditons (2.12) and (2.14) are satisfied. Both inequalites of (2.12) lead to a bound for $k$ as given by (2.1), while (2.14) gives about twice that. The lower bound for $|B|$ is case $j=k$ of (2.5).

3. From structure to minimum. In this section we show a result that goes in the opposite direction to Lemma 2.1.

LEMMA 3.1. Let $A$ be a finite set of integers, symmetric about the origin and not containing 0 . Put

$$
f(x)=\sum_{a \in A} e^{2 \pi i a x} .
$$

Suppose that there are sets $S, T$ of integers, an integer $d \neq 0$ and an even positive integer $L$ such that $|S|,|T| \geq L$ and $S+T+\{0, d\} \subset A$. Then

$$
\min f(x)<-\sqrt{L} / 2 .
$$

Proof. First we establish the existence of finite sets of integers $U, V$ such that $|U|,|V| \geq L / 2, U-V \subset A, U \subset A, V \cap A=\emptyset$ and $0 \notin V$. 
Assume $0 \in T$ (this can be achieved by shifting $S$ and $T$ if necessary), and write

$$
r_{j}=|(S-j d) \cap A| .
$$

We have $r_{0} \geq L$ and $r_{j}=0$ for large $j$, so there is a $j$ such that $r_{j} \geq L / 2$ $>r_{j+1}$. Write $A_{0}=A \cup\{0\}$.

Now if

$$
|(j d-T) \cap A|<L / 2,
$$

then put

$$
U=(S+j d) \cap A, \quad V=(j d-T) \backslash A_{0} .
$$

We have

$$
\begin{aligned}
& |U|=r_{j} \geq L / 2, \\
& |V|=|T|-|(j d-T) \cap A|-1>|T|-L / 2-1 \geq L / 2-1
\end{aligned}
$$

and

$$
U-V \subset(S+j d)-(j d-T)=S+T \subset A .
$$

If (3.2) does not hold, then we put

$$
U=(j d-T) \cap A, \quad V=(S+(j+1) d) \backslash A_{0} .
$$

We have $|U| \geq L / 2$ by the negation of (3.2), and

$$
\begin{gathered}
|V|=|S|-r_{j+1}-1>L / 2-1, \\
U-V \subset(j d-T)-(S+(j+1) d)=-(S+T+d) \subset-A=A .
\end{gathered}
$$

We define $K$ by $\min f(x)=-K$, and another function $h$ by

$$
h(x)=\frac{1}{|U|} \sum_{u \in U} e(u x)-\frac{1}{|V|} \sum_{v \in V} e(v x) .
$$

We write simply $\int$ for $\int_{0}^{1} \ldots d x$.

Observe that we know $0 \notin V$, and also $0 \notin U$ by $U \subset A$, hence $\int h(x)=0$. We have $\int h(x) f(x)=1$ since $U \subset A, V \cap A=\emptyset$, thus

$$
\int h(x)(f(x)+K)=1 .
$$

Furthermore

$$
\begin{aligned}
|h(x)|^{2}= & |U|^{-2} \sum_{u, u^{\prime} \in U} e\left(\left(u-u^{\prime}\right) x\right)+|V|^{-2} \sum_{v, v^{\prime} \in V} e\left(\left(v-v^{\prime}\right) x\right) \\
& -(|U||V|)^{-1} \sum_{u \in U, v \in V}(e((u-v) x)+e((v-u) x)) .
\end{aligned}
$$

As always $u-v, v-u \in A$, we see that

$$
\int|h(x)|^{2} f(x) \leq 1+1-2=0 .
$$


Since clearly

$$
\int|h(x)|^{2}=|U|^{-1}+|V|^{-1} \leq 4 / L
$$

we have

$$
\int|h(x)|^{2}(f(x)+K) \leq 4 K / L .
$$

By Cauchy's inequality and (3.3) we have

$$
\begin{aligned}
1 & =\int h(x)(f(x)+K) \leq\left(\int|h(x)|^{2}(f(x)+K)\right)^{1 / 2}\left(\int(f(x)+K)\right)^{1 / 2} \\
& \leq(4 K / L)^{1 / 2} K^{1 / 2},
\end{aligned}
$$

that is, $K \geq \sqrt{L} / 2$ as claimed.

4. Completion of the proof and remarks. We prove Theorem 2.

Let $\min f(x)=-K$. By Lemma 2.1, with $k$ defined by (2.1), there are integers $\beta_{1}, \ldots, \beta_{k}$ and a set $B$ such that always $\sum \varepsilon_{j} \beta_{j}+B \subset A$. Put $S=B$,

$$
T=\left\{\sum_{j=1}^{k-1} \varepsilon_{j} \beta_{j}\right\}
$$

and $d=\beta_{k}$. We have $S+T+\{0, d\} \subset A$ and $|S|>|T|=2^{k-1}$, so an application of Lemma 3.1 yields

$$
K \geq 2^{(k-1) / 2} \geq \exp \left\{\frac{\log 2}{2} \frac{\log n}{4 \log K+c_{6}}-2\right\} .
$$

After taking the logarithm and rearranging this yields a quadratic inequality for $\log K$ and by a simple calculation we find the bound of the theorem.

Concluding REMARKs. The proof of Lemma 2.1 closely follows Bourgain's argument from [2]. We wrote it in detail, since there is no statement in his paper which we could immediately apply. He proves the existence of a more complicated structure, namely a cube with sides of 5 in contrast to our 2, that is, a set of the form

$$
\left\{b+\sum \varepsilon_{i} \beta_{i}: \varepsilon_{i} \in\{0, \ldots, 4\}\right\} .
$$

This makes the argument more involved and reduces the size of $k$. This part of the proof is somewhat simpler in this paper than in Bourgain's, but this simplification is due to the fact that we are content with the simpler structure (2.3) rather than (4.1), and there is no essential new idea here.

The improvement is in the second part, where we can make use of the simpler set (2.3). Though the second part is rather different from the approach in [2], I acknowledge that it also was motivated by analyzing Bourgain's argument. 
We remark that since the reciprocals of the cardinalities occur in (3.4) it does not help that one of the sets $U, V$ (or $S, T$ ) is larger. The assumption $U-V \subset A$ could be weakened to a condition asserting that most differences $u-v$ are in $A$; however, in this case one can find somewhat smaller subsets $U^{\prime} \subset U, V^{\prime} \subset V$ such that $U^{\prime}-V^{\prime} \subset A$.

Acknowledgements. I profited much by discussing this problem with Antal Balog.

\section{References}

[1] J. Bourgain, Sur le minimum de certaines sommes de cosinus, Publ. Math. Orsay 84-01 (1984), no. 2, 7 pp.

[2] _, Sur le minimum d'une somme de cosinus, Acta Arith. 45 (1986), 381-389.

Alfréd Rényi Mathematical Institute

Hungarian Academy of Sciences

Budapest, Pf. 127

H-1364 Hungary

E-mail: ruzsa@renyi.hu 